\title{
COMPORTAMIENTO Y PERFIL DE LOS CONSUMIDORES DE SERIES DE FICCIÓN Y OTROS PRODUCTOS AUDIOVISUALES EN EL SIGLO XXI.
}

Noelia Araújo-Vila1. Universidad de Vigo. España. naraujo@uvigo.es

José Antonio Fraiz-Brea. Universidad de Vigo. España. jafraiz@uvigo.es

\section{RESUMEN}

El estudio del comportamiento del consumidor es un campo compartido por diversas disciplinas, fruto del interés por conocer los gustos, preferencias o rasgos que lo definen. El sector audiovisual, tras su apogeo en las últimas décadas, también ha mostrado interés en conocer cuál es el perfil de su consumidor, el espectador, o cuáles son las pautas que sigue a la hora de consumir uno u otro producto de este sector. Por ello, el presente artículo se centra en posibles variables que definen al espectador y en factores que influyen en él a la hora de consumir un producto audiovisual.

PALABRAS CLAVE: Consumidor audiovisual - Perfil - Influencia - Sector audiovisual.

\section{BEHAVIOR AND PROFILE OF FITCION SERIES AND OTHER AUDIOVISUAL PRODUCTS CONSUMER IN 21TH CENTURY.}

\section{ABSTRACT}

The study of consumer behavior is an area shared by different disciplines, the result of interest to know the tastes, preferences or traits that define it. The audiovisual

\footnotetext{
${ }^{1}$ Autor correspondiente

Noelia Araújo-Vila: Profesora del Máster en Dirección y Planificación del Turismo Interior y de Salud, Facultad de Ciencias Empresariales y Turismo, Universidad de Vigo. España.

Correo: naraujo@uvigo.es
} 
sector, after its peak in recent decades, has also shown interest in knowing what is the profile of its consumer, the viewer, or what are the guidelines that follow when consuming one or another product of this sector. Therefore, this research focuses on possible variables that define the audience and factors that influence it at the time of consuming an audiovisual product.

KEY WORDS: Audiovisual consumer - Profile - Influence - Audiovisual.

\section{INTRODUCCIÓN}

\subsection{El consumidor}

Independientemente del sector que se esté estudiando, el punto de partida en lo que al comportamiento del consumidor se refiere es el mismo, "el conjunto de actividades que realizan las personas cuando seleccionan, compran, evalúan y utilizan bienes y servicios, con el objeto de satisfacer sus deseos y necesidades, actividades en las que están implicados procesos mentales y emocionales, así como acciones físicas" (Wilkie, 1994); radicando la diferencia en el tipo de carencia o necesidad, en el bien o servicio a consumir para satisfacerla y en las actividades que se llevan a cabo para adquirirlo y consumirlo. Podemos por tanto resumir las pautas de comportamiento de un consumidor a través de tres características (Mollá y Berenguer, Gómez y Quintanilla, 2006):

Incluir numerosas actividades, que preceden, acompañan y siguen tras la compra, pudiendo resumirlas en tres etapas:

a)Precompra: detección de la necesidad, búsqueda de información y evaluación de posibles alternativas.

b)Compra: selección del lugar de compra y del producto/servicio.

c) Postcompra: utilización del producto/servicio y equiparación con las expectativas, lo que deriva en satisfacción, o carencia de la misma insatisfacción-.

- Ser una conducta motivada: el punto de partida del consumo es el descubrimiento de una necesidad, la cual es satisfecha para dar respuesta a diversos estímulos, por ejemplo situacionales -un anuncio publicitario- o personales. Lo imprescindible es la existencia de una motivación que desemboque en el consumo.

- Poner en funcionamiento el sistema psicológico del individuo: durante el comportamiento de compra el individuo pondrá en marcha alguna o varias variables psicológicas (cognitivas, afectivas o conductuales), dependiendo del producto/bien a comprar o incluso a la situación anímica del momento. 


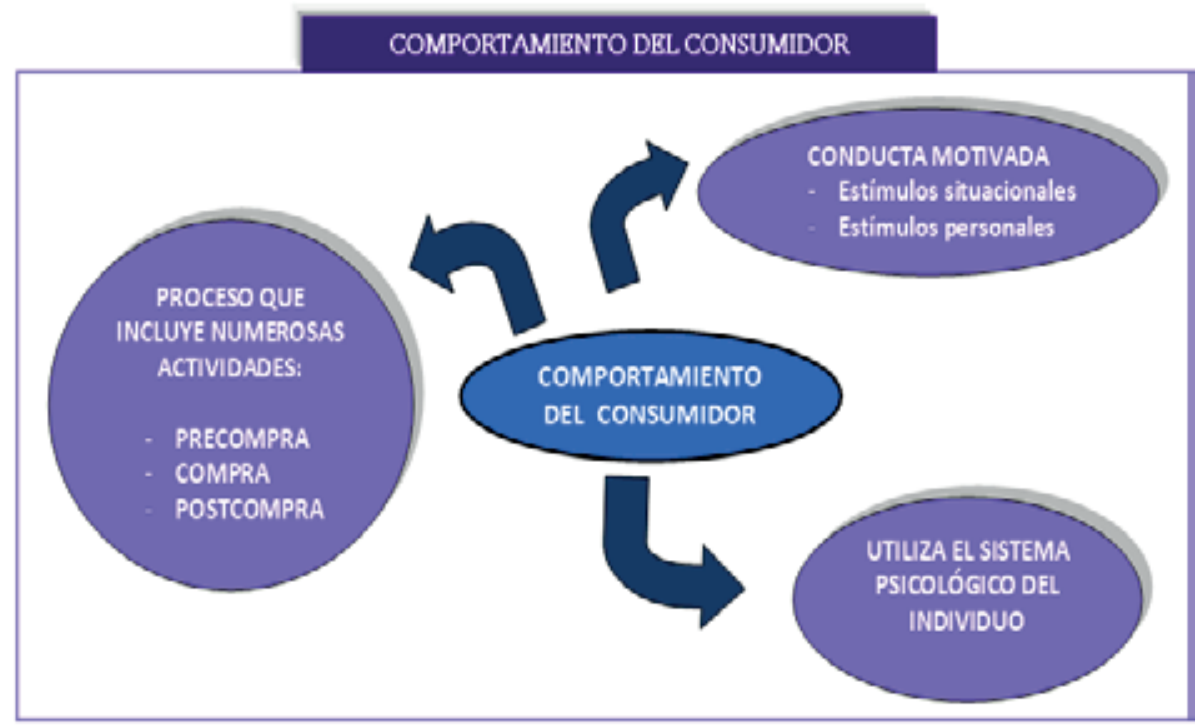

Figura 1. Características del comportamiento del consumidor

Fuente: elaboración propia a partir de Mollá, Berenguer, Gómez y Quintanilla (2006)

Una vez conocidas las características que forman parte del comportamiento de un consumidor, es decir, el proceso que le lleva a actuar de una $u$ otra forma ante la adquisición de un bien/producto, el siguiente paso es centrarse precisamente en aquellas necesidades, motivaciones y perfiles de consumidor del sector objeto de estudio, el audiovisual, para así conocer qué productos son los adecuados ante las necesidades, deseos y gustos del espectador. Dichas variables serán analizadas en el presente artículo, partiendo de estudios clásicos como el de Frank, Massy y Wind (1972), que analizaba a los consumidores en función de la edad, el género, la ubicación geográfica, personalidad, actitud o estilo de vida.

\section{DESARROLLO}

\subsection{El Consumidor Audiovisual}

En lo que respecta al consumidor audiovisual, el subsector más estudiado es el del cine, presentándose un consumidor cinematoFigura que ha sido ya abordado desde distintas disciplinas, como la psicología, la comunicación o el mundo del ocio (Eliashberg y Shugan, 1997; Hirschman, 1987), lo que ha derivado también en el interés por otros productos de este mismo sector, como la televisión (Hirschman, 1987). Para analizar dicho sector, se parte de nuevo de las teorías generalistas en lo que a comportamiento del consumidor se refiere, es decir, conocer cuál es el procesamiento de información que realiza el individuo, las etapas de resolución de problemas que desembocan en una decisión final sobre la compra de uno u otro producto (Foxall, Goldsmith y Brown, 2002).

Hasta la década de 1990, existía un gran vacío respecto a esas pautas, pensamientos y comportamientos del consumidor audiovisual, por lo que Linton y Petrovich (1988) tras detectar esa falta de conocimientos, inician una investigación centrándose en el consumidor cinematoFigura (Cepeda, 2005, p.91). Tras su investigación concluyen, 
que al igual que cualquier otro producto o bien, en el cine el consumidor se centra en tan sólo algunos atributos de la película, ya que la capacidad de asimilación y procesamiento humano es limitada; es decir, el escoger una u otra película viene determinado por tan sólo algunas características, no todas, sino aquéllas que el consumidor considera determinantes.

Tras estas conclusiones iniciales, se llevaron a cabo otros estudios, predominando los correlacionales y experimentales, y en menor medida cualitativos. Así se lograron avances que explicaron en mayor profundidad factores influenciadores en el comportamiento del consumidor cinematoFigura, como el de Neelameghan y Jain (1999), que demostró que la elección de una película depende de la información directa que se tiene sobre el producto (avisos, comentarios de críticos, boca-oído) y de las expectativas sobre la estimulación emocional y el interés latente en el producto. Otros ejemplos son los siguientes (Cepeda, 2005, p.94-95):

- Diseños correlaciónales:

a) Relación entre los comentarios críticos de una película y desempeño en taquilla, obteniéndose correlación positiva entre ambas variables (Eliashberg y Shugan, 1997).

b)Relación entre búsqueda de sensaciones y películas de terror (Edwards, 1991).

c) Relación entre la gratificación buscada en una película y asistencia (Palmgreen y Lawrence, 1991).

- Diseños experimentales: mediante uso de aparatos mecánicos que según se pulsase uno u otro botón durante el visionado de una película indicaban agrado o desagrado de esa escena o escenas. Si no se oprimía ningún botón en algún momento era señal de indiferencia (Eliashberg y Sawhey, 1994).

Dentro del sector audiovisual, la variedad de subsectores, y dentro de cada subsector, de productos, es elevada, pudiendo satisfacer así a un mayor número de segmentos y perfiles de consumidores. Por lo tanto analizando diversas variables, como pueden ser el sexo, edad, la ubicación geográfica, personalidad, actitud o estilo de vida (Frank, Massy y Wind, 1972), obtenemos distintos perfiles de consumidores dentro de un mismo sector, ya que el sector audiovisual "permite el encuentro con personas que representan ámbitos sociales, étnicos o culturales diversos" (Medrano, Cortés, Aierbe y Orejudo, 2010, p.4), por lo que resulta imposible hablar de un consumidor audiovisual tipo.

A continuación se analizan algunas variables para establecer diferentes grupos, y observar cuáles son las actividades, motivaciones, necesidades o medios de acceso a la información sobre el producto deseado de cada uno de ellos, partiendo de las investigaciones citadas y alguna posterior.

\subsection{Edad}

Independientemente de la edad, el consumidor de productos audiovisuales tiende a 
buscar películas, series $\mathrm{u}$ otro tipo de programas que reflejen sus problemas e inquietudes (França, 2001; Montero, 2006; Oliver, 2002). En cuanto a lo que a consumo de televisión se refiere, no se encuentran realmente diferencias excesivas entre adolescentes, jóvenes y adultos y la franja horaria favorita para consumir este medio es de 21 a 24 horas (Medrano et al, 2010, p.4). En el campo del cine, los resultados son similares, no variando el grado de disfrute de una película entre hombres y mujeres o distintas edades (Eliashberg y Sawhney, 1994). Aún así, las posibles diferencias radicarán en que dependiendo del momento de la vida de un individuo, las inquietudes irán variando, así por ejemplo la mayoría de los adolescentes buscan en la cartera de productos del sector audiovisual contenidos positivos, los jóvenes la evasión y los adultos/padres la evasión, estrechamente seguida de la información y actualidad (Medrano et al, 2010, p.11; estudio realizado a 859 individuos del País Vasco, correspondientes a tres grupos de edad) (Tabla 1). Éstas serían por tanto, parte de las motivaciones, es decir, parte del proceso de la precompra del producto. En cuanto a la búsqueda de información la variable edad es también un elemento clave, ya que el acceso mayoritario a la información en los individuos adolescentes y jóvenes es Internet y las nuevas tecnologías, predominando foros, webs o redes.

Tabla 1. Preferencias en consumo de productos audiovisuales según grupos de edad

\begin{tabular}{|l|c|c|c|c|c|}
\hline Categorías & \multicolumn{2}{c}{ SEXO } & \multicolumn{3}{c|}{ GRUPO DE EDAD } \\
\hline $\begin{array}{l}\text { Tipo de Programa/edad } \\
\text { Evasión }\end{array}$ & Mujer & Hombre & Adolescentes & Jóvenes & Adultos/ Padre \\
\hline Diversión/humor & $22,3 \%$ & $17,4 \%$ & $14,4 \%$ & $32,7 \%$ & $36,4 \%$ \\
\hline Formación/actualidad & $18 \%$ & $23,8 \%$ & $24,2 \%$ & $19,8 \%$ & $4,1 \%$ \\
\hline Contenidos & $9,1 \%$ & $6,40 \%$ & $0,40 \%$ & $1 \%$ & $33 \%$ \\
\hline Contenidos positivos & $38,6 \%$ & $40,4 \%$ & $48,6 \%$ & $27,7 \%$ & $6,6 \%$ \\
\hline Otros & $7,3 \%$ & $5,2 \%$ & $5,3 \%$ & $5,9 \%$ & $11,6 \%$ \\
\hline
\end{tabular}

Fuente: elaboración propia a partir de Medrano et al. (2010, p.11)

\subsection{Género}

En lo que al género se refiere, se encuentran conductas diferenciadas ligadas también en ciertos aspectos a la cultura y educación de cada grupo social, así en Norteamérica las chicas jóvenes ven menos televisión que los chicos de la misma edad y son más conscientes de ciertas conductas negativas transmitidas vía televisión. Otros autores, tras diversos estudios, reflejan otros rasgos diferenciadores, como la preferencia de contenidos violentos y deportivos por parte de los chicos (Mustonen y Pulkinen, 1997; Watt y Krull, 1977; França, 2001) y programas de romance, humor y entretenimiento por parte de las chicas (França, 2001). Sin embargo Horton y Arquette (2000), tras analizar a jóvenes hispanos, ingleses y norteamericanos, en lo que respecta a programas de humor y drama, no encuentran diferencias significativas de género. Además de las preferencias en cuanto a los contenidos, también se establecen diferencias en cuanto a la credibilidad de lo visionado o el impacto que provoca sobre el individuo, así en el caso de las series, las chicas otorgan 
mayor credibilidad a las mismas que los chicos, tal vez por la empatía establecida con los personajes (Mestre, Pérez-Delgado, Samper y Martí, 1998; Sánchez-Queija, Oliva y Parra, 2006; Seiter, 1989); y en el caso de la televisión los hombres están más expuestos al impacto de ésta (del Río, Álvarez y del Río, 2004).

Volviendo a un caso nacional (estudio realizado por Medrano et al. en el País Vasco), no se encuentran notables diferencias en las preferencias respecto al tipo de contenido de hombres a mujeres, siendo los contenidos positivos (de los items tratados en la Tabla 1) los preferidos por la mayoría de los hombres $(40,4 \%)$ y de las mujeres $(38,6 \%)$.

\subsection{Actitud}

Entendemos por actitud "la disposición de ánimo manifestada de algún modo" (RAE), es decir, en lo que respecta a comportamiento del consumidor, la predisposición a consumir uno $\mathrm{u}$ otro producto en función del estado de ánimo circunstancial. Esto resulta clave en la elección de un producto audiovisual, tanto en la fase de precompra, en la que se evalúan las alternativas, como en la de postcompra.

Los consumidores/espectadores tienden a seleccionar dentro de la industria de entretenimiento, y dentro de ésta, de la audiovisual, aquellos productos que sirven para mantener y maximizar su placer y minimizar su dolor (Bryant y Miron, 2002; Zillmann, 1988). Todo ello se transforma en una relación directa en la elección de un producto, por ejemplo una película, ya que una persona momentáneamente triste evitará películas trágicas o dramáticas (Winoto y Tang, 2010, p.6086), o un capítulo de una serie, ya que en caso de cansancio o agotamiento psíquico se decantará por el humor o la comedia.

Del mismo modo que el ánimo influye en la compra, también lo hace en la postcompra, ya que los espectadores con un estado de ánimo positivo evaluarán de manera más favorable el producto que los que presentan un estado de ánimo negativo (Gardner, 1985; Veithc y Griffitt, 1976).

Se concluye así, que de modo consciente o inconsciente, los consumidores de productos vinculados con la industria del entretenimiento, intentan elegir aquello que en términos de intensidad y duración potencie el estado de ánimo positivo y disminuya el dolor (Gardner, 1985; Holbrook y Gardner, 2000; Isen, 1984; Oliver, 2008; Ryan, Rigby y Przybylski, 2006).

\subsection{Personalidad y empatía con los personajes de ficción}

Uno de los factores clave a la hora de influir en la elección de uno u otro producto audiovisual es la personalidad, gusto y preferencias de cada individuo -variables subjetivas del modelo de Frank, Massy y Wind (1972)-, lo que va a llevar a empatizar con unos u otros personajes de la ficción. Así en España, referenciando de nuevo el 
estudio de Medrano et al., 2010, los personajes preferidos por los espectadores son los de series cómicas $(27,7 \%)$, seguidos de dibujos animados $(13,90 \%)$ y algo más de lejos los humorísticos $(13,03 \%)$ y de telenovelas $(5,7 \%)$. Los motivos que llevan a elegir uno $u$ otro personaje, son principalmente el ser divertido $(36,63 \%)$, la profesionalidad $(20,37 \%)$ u otras cualidades físicas socialmente estimadas como positivas $(12,20 \%)$. Datos del mismo estudio, muestran las posibles relaciones entre el género y edad del individuo y la elección del género y edad del personaje de ficción, siendo los resultados que independientemente del género, los personajes más valorados o preferidos son los masculinos y en lo que respecta a la edad independientemente del grupo de edad del individuo, los personajes favoritos son los adultos $(80,3 \%)$ (Tabla 2$)$.

Tabla 2. Características de los personajes de productos audiovisuales preferidos por los consumidores

\begin{tabular}{|c|c|c|c|c|c|c|c|}
\hline \multirow[t]{2}{*}{ CATEGORÍAS } & \multicolumn{2}{|c|}{ SEXO } & \multicolumn{5}{|c|}{ GRUPO DE EDAD } \\
\hline & Mujer & Hombre & & lescents & & óvenes & Adultos \\
\hline \multicolumn{8}{|c|}{ TIPO DE PROGRAMA (\%) } \\
\hline Series policíacas & 5,7 & 2,9 & & 4,1 & & 5,8 & 5,8 \\
\hline Series cómicas & 36,1 & 22,1 & & 31,5 & & 20,9 & 30,8 \\
\hline Series de oficios & 3,2 & 5,1 & & 2,1 & & 16,3 & 1,9 \\
\hline Deportes & 0,3 & 2,6 & & 1,4 & & 0 & 1,9 \\
\hline Dibujos animados & 10,6 & 35,7 & & 25,1 & & 12,8 & 3,8 \\
\hline Telenovelas & 16 & 2,9 & & 12,4 & & 4,7 & 0 \\
\hline Concursos & 3,4 & 2,2 & & 1,9 & & 5,8 & 7,7 \\
\hline Humor & 10 & 14,7 & & 11,6 & & 14 & 13,5 \\
\hline Otros & 3,7 & 1,8 & & 2,3 & & 5,8 & 3,8 \\
\hline Series generales & 3,4 & 4,4 & & 3,9 & & 2,3 & 5,8 \\
\hline Prensa rosa & 3,4 & 3,3 & & 2,3 & & 5,8 & 9,6 \\
\hline Culturales e informativos & 4 & 2,2 & & 1,4 & & 5,8 & 15,4 \\
\hline \multicolumn{8}{|c|}{ MOTIVACIÓN (\%) } \\
\hline \multicolumn{3}{|l|}{ Diversión } & 33,6 & 50,2 & 42,4 & 35,4 & 32,1 \\
\hline $\begin{array}{l}\text { Otras cualidades físicas } \\
\text { valoradas }\end{array}$ & \multicolumn{2}{|c|}{ positivamente } & 16 & 4,7 & 13,8 & 2,5 & 1,9 \\
\hline No convencional & & & 2,6 & 4,3 & 2,8 & 6,3 & 3,8 \\
\hline Profesionalidad & & & 17,7 & 11,1 & 12,5 & 20,3 & 28,3 \\
\hline Personalidad positiva & & & 13,1 & 6,7 & 9,5 & 13,9 & 13,2 \\
\hline Características negativas & & & 6,3 & 14,6 & 10,8 & 5,1 & 7,5 \\
\hline Características positivas & & & 6,8 & 6,3 & 5,7 & 11,4 & 7,5 \\
\hline Identificación personal & & & 4 & 2 & 2,5 & 5,1 & 5,7 \\
\hline
\end{tabular}




GÉNERO Y EDAD DEL PERSONAJE (\%)
\begin{tabular}{|l|l|l|l|l|l|}
\hline Mujer & 25,2 & 10,6 & 16,1 & 25,3 & 33,3 \\
\hline Hombre & 74,8 & 89,4 & 83,9 & 74,7 & 66,7 \\
\hline Infancia & 6,2 & 7,7 & 8,1 & 2,5 & 2,1 \\
\hline Adolescencia & 25,1 & 6,2 & 20,4 & 2,5 & 4,2 \\
\hline Madurez & 63,5 & 84,2 & 68,6 & 91,1 & 81,2 \\
\hline Tercera Edad & 5,3 & 1,9 & 2,9 & 3,8 & 12,5 \\
\hline NATURALEZA DEL PERSONAJE (\%) & 82,3 & 87,1 & 88,9 & 73,4 & 60,8 \\
\hline Ficción & 17,7 & 12,9 & 11,1 & 26,6 & 23,2 \\
\hline Realidad
\end{tabular}

Fuente: elaboración propia a partir de Mendano et al. (2010, p.13-15)

Podemos concluir, que aunque la diversidad de perfiles dentro del sector audiovisual es heterogénea, se encuentran ciertas coincidencias entre distintos colectivos (segmentados por edad y sexo), como la preferencia por personajes de series cómicas, de sexo masculino y edad adulta que no son reales $\mathrm{y}$, la diversión, como factor motivador para elegir el producto a consumir.

Por ello, las cadenas de televisión nacionales, conscientes de tales preferencias apuestan por programación que cumpla tales expectativas, como son el caso de dos cadenas de reciente creación en el panorama televisivo español, La Sexta y Cuatro, contando las dos con mayor apoyo masculino, apostando por las series de ficción, documentales y reporterismo. Cuatro se define a sí misma como una cadena destinada a un público joven, urbanita y de clase media-alta, y La Sexta a un público de clase social más moderada y joven (Vaca, 2010).

\subsection{Influencias en el comportamiento del consumidor audiovisual}

Independientemente del tipo del consumidor ante el que nos encontramos, la conducta de éste puede estar dirigida o afectada por una serie de variables externas al mismo, las cuales deben de ser analizadas para comprender el proceso de compra de uno $u$ otro producto o servicio (Alonso y Grande, 2004, p.105), ya sea audiovisual o de cualquier otro sector.

Dentro de estas variables se encuentran los denominados factores del entorno, entre los que encontramos los diferentes grupos de pertenencia de los individuos o la cultura y estratos sociales hasta influenciadores de menor cuantía como grupos referenciales (amigos y familia) (Alonso y Grande, 2004, p.105).

\subsubsection{Factores influenciadores en el consumidor}

En toda investigación sobre el comportamiento del consumidor el objetivo final es dar respuesta a una serie de preguntas, las cuales se intentan resolver a través del análisis del ambiente que rodea al consumidor. Estas cuestiones que desencadenan el 
interés por el conocimiento del entorno, las podemos resumir en las 7 O's propuestas por Kotler (2000), las cuales pretenden dar espuesta a las preguntas más importantes sobre cualquier mercado (Tabla 3 ).

Tabla 3. Siete O's del mercado

\begin{tabular}{|l|c|}
\hline ¿Quién constituye el mercado? & OCCUPANTS \\
\hline ¿Qué compra el mercado? & OBJECTS \\
\hline ¿Por qué compra el mercado? & OBJETIVES \\
\hline ¿Quién participa en la compra? & ORGANIZATIONS \\
\hline ¿Cómo compra el mercado? & OPERATIONS \\
\hline ¿Cuándo compra el mercado? & OCCASIONS \\
\hline ¿Dónde compra el mercado? & OUTLETS \\
\hline
\end{tabular}

Fuente: Kotler (2000)

Los pioneros en buscar dichos datos, y por tanto analizar el perfil y comportamiento del consumidor, fueron los investigadores de marketing, tales como Scott en los años 1930 o Howard y Sheth en 1969, no siendo hasta la década de 1970 cuando empiezan los estudios en el sector servicios. Tras todos estos estudios, se llega a un modelo que clasifica los factores influenciadores en el consumidor, entre los que encontramos los de entorno y personales (Beckman y Gilson, 1978: Nicosia, 1966; Miller y Grush, 1983; Howard y Sheth, 1969; Rother, 1965 y 1992 y Bettman y Morgan, 1972).

Entendemos por entorno los factores culturales, sociales, personales o psicológicos. A continuación se detallarán los factores del entorno y personales, agentes que inciden directamente en la conducta del consumidor independientemente de la relación producto- mercado.

\subsubsection{Factores de entorno y personales}

Nos estamos refiriendo por tanto a los influenciadores que forman parte del proceso de formación de una opinión previa a la compra, es decir, agentes de las dimensiones personales y sociales de un individuo, ajenos en todo momento al intercambio propio de la compra o consumo (de Borja, Casanovas y Bosch, 2002, p.54). Podemos resumirlos, desde un efecto menos directo a uno más directo, en cultura, estratos sociales, grupos de referencia y familia (Schnake, 1988) (Figura 2).

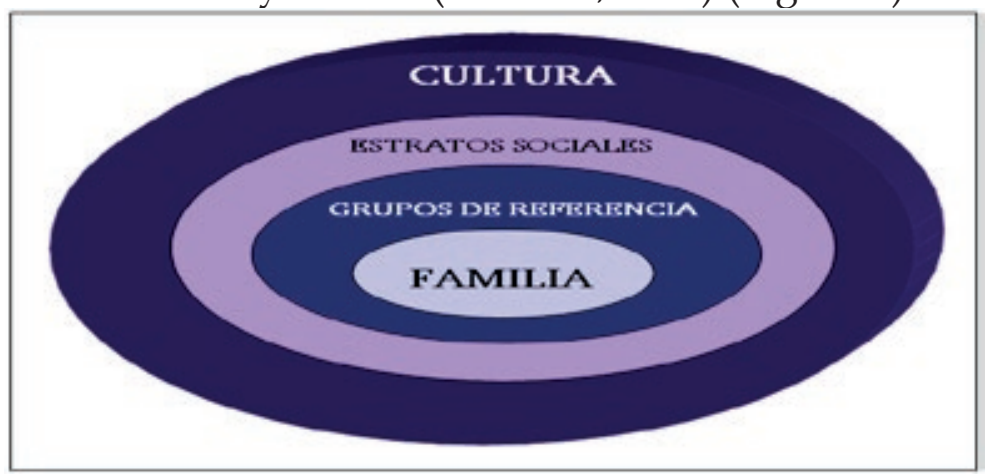

Figura 2. Agentes influenciadores en el comportamiento del consumidor del entorno Fuente: elaboración propia a partir de Schnake (1988) 


\section{Cultura}

El concepto de cultura ha sido estudiado por diversas disciplinas y definido por diversos autores, pudiéndolo resumir como el conjunto de pautas, creencias o conductas adquiridas por el hombre como parte integrante de la sociedad (Benedict, 1934; Kretch y Crutchield, 1952; Linton, 1968; Sherry, 1986; Tylor, 1913).

Centrándonos en el sector investigado, el audiovisual, la influencia de la cultura es un factor que no se puede obviar, el cual va a condicionar qué productos, y dentro de éstos qué tipo, van a ser demandados y consumidos. Así por ejemplo, en Norteamérica, la cultura y moral transmitida, hace que la población sea más estricta con los contenidos televisivos visionados por las chicas adolescentes, lo cual va a limitar los productos ofertados y el consumo de los mismos por este sector dentro del campo de la televisión (Greenberg, Rampoldi y Ver, 1998). Otro claro ejemplo, es el caso de Bollywood, donde la tradición y cultura del país indio ha dado lugar a este género cinematoFigura, el cual está basado en Mumbai y se ha convertido en la cultura dominante de la India moderna (Mishra, 2009, p.439).

\section{Estratos sociales}

Al igual que el concepto de cultura, el de estratos sociales posee varias definiciones dependiendo del autor que lo ha estudiado, pudiendo resumirlas en individuos pertenecientes a un grupo relativamente homogéneo, es decir, con características, actitudes y estilos de vida similares (Alonso y Grande, 2004; Engel, Kollat y Blackwell, 1973 y León y Olabarría, 1991).

Así, aproximándonos al sector que nos ocupa, en lo que al entretenimiento se refiere, la televisión local -gratuita- es el medio más usado por estratos sociales medio y bajos (en términos económicos), mientras que presenciar una película en un teatro de cine, -lo cual supone comprar una entrada de coste elevado y un desplazamiento hasta el teatro-, será consumido por estratos con un mayor nivel adquisitivo y nivel de vida más elevado, en los que este tipo de entretenimiento está considerado como habitual dentro de sus intereses y valores (Cepeda, 2005, p.91).

\section{Grupos de referencia}

Además de la cultura y el estrato social, los individuos se ven condicionados por grupos más próximos con los que se relacionan, estando así dirigidos habitualmente por las acciones del grupo (Sherif y Cantril, 1974). Se define por tanto el grupo de referencia como "el conjunto de individuos que sirve como punto de comparación para una persona en la formación de sus valores generales o específicos, actitudes o comportamientos" (Foxall, Goldsmith y Brown (2002).

Ejemplos de ello en el sector del ocio es la proliferación de las redes sociales, dentro de las cuales, las generalistas reparten su orientación entre el entretenimiento y la información de interés valorada y comentada, buscando los individuos pertenecer a 
las mismas y ser las propias fuentes de tal información y referencia para otros usuarios (Campos, 2008, p.287). Dentro de las redes sociales también ha ido en aumento el número de grupos, especialmente en facebook, buscando así contacto con individuos con los mismos intereses -psicogrupos- o pertenecer a organizaciones sociales -grupos secundarios- (Alonso y Grande, 2004). Entre éstos y aproximándonos así al sector audiovisual, han emergido diversos de ellos vinculados a series, como el de Lost (con 4.842.074 seguidores), Grupo oficial de Heroes (1.872.028) o el de The Big Bang Theory (3.172.870 seguidores) (Facebook, 2010), o a nivel español ejemplos como la serie Física o Química, con 109.379 seguidores, o El Internado con 183.955 usuarios (Facebook, 2010).

\section{Familia}

Los individuos de la familia son el grupo de pertenencia básico y primario de un individuo, siendo el que crea mayor influencia en lo que respecta al comportamiento de un consumidor (Kotler, 2000; Eshleman, 2000), ya que es el grupo que aporta al individuo las normas, valores, creencias y conductas que lo orientan (Alonso, 1984).

Al hablar de familia, se debe de tener en cuenta la evolución de dicho concepto, pasando de ser marido, esposa e hijos, al concepto aumentado de familias monoparentales o familias ampliadas (matrimonios en segundas nupcias), lo cual también ha hecho evolucionar los valores e influencias inculcados por las mismas (de Borja et al, 2002, p.64). Por tanto, la influencia de la familia va a ser variada, dependiendo del tamaño, de los valores, normativa o información de la misma (Borja et al, 2002, p.65), aunque siempre se van a dar una serie de roles entre los distintos miembros: informador, influyente o experto, decisor, comprador y consumidor (Assael, 1987).

Así, en lo que se refiere a los jóvenes españoles (14-25 años), se parte de la premisa de que éstos son los usuarios y consumidores de los nuevos soportes tecnológicos de acceso al sector audiovisual (Internet o móviles entre otros), (López, 2009), siendo ya en 2007 el 70\% de éstos los que habían descargado en alguna ocasión contenidos audiovisuales (I Encuentro sobre Televisión Online de Televeo, 2007). Por tanto los jóvenes también se han convertido a la vez en iniciadores e influenciadores de la unidad familiar, ya que reclaman contenidos de pago (sea tarifa telefónica, televisión de pago...) (López, 2009), y van a depender de la disposición al pago de sus padres, que desempeñarán el rol de decisores y en ocasiones compradores.

En otras situaciones, los miembros de las familias se vuelven los influenciadores más directos, bien siendo estrictos en los contenidos visionados por sus hijos -como pasa con los padres norteamericanos, los cuales tienden a controlar y limitar en mayor cuantía los contenidos visionados por sus hijas que los de sus hijos (Greenberg, Rampoldi y Ver, 1998)-, bien meramente aportando su opinión sobre cierto programa o producto audiovisual, ya que la familia es el grupo de referencia más importante en funciones como el filtrado de información (Alonso y Grande, 2004, p.260). 


\section{CONCLUSIONES}

El investigar y conocer las pautas de consumo de los individuos es objeto de interés de diversos sectores y campos, ya que el éxito o fracaso de una industria, radica precisamente en saber cubrir las necesidades del mercado y ofertar productos y servicios que satisfagan o incluso superen las expectativas creadas. Por ello, el sector audiovisual, componente de la industria del ocio en pleno apogeo a día de hoy, no ha sido menos y también ha realizado diversas investigaciones en esta materia. Aparece así, el denominado consumidor audiovisual, del cual hasta pasado el año 1990 existía un gran vacío. Es a partir de esta década que aparecen estudios que intentan limitar variables que lo definan, como la edad, el género, la actitud o la personalidad, todo ello con el fin de determinar cómo es este consumidor y saber qué espera o demanda en el sector audiovisual. En pleno siglo XXI, nos encontramos ante un consumidor, o mejor dicho, espectador, con un perfil muy heterogéneo, con diversidad de gustos, edades o preferencias, lo que dificulta hablar de un estándar. Podemos hablar de unas tendencias mayoritarias, como el interés por series cómicas o dibujos animados, o una marcada preferencia por los contenidos audiovisuales que provoquen diversión.

Es evidente el fin entretenedor de todos los productos del sector audiovisual, el cual sí se está persiguiendo, pero no podemos establecer una serie de productos limitados, sino que es necesaria una variada oferta audiovisual que se adapte a los distintos segmentos de mercado con los que cuenta este sector. En concordancia con tales resultados, podemos citar a modo de ejemplo la aparición de la televisión digital, la cual amplía las posibilidades de crecimiento y acceso a un mayor público con diferentes preferencias y características, para todas las cadenas televisivas actuales, pudiendo así satisfacer a un mayor número de potenciales espectadores o consumidores audiovisuales.

\section{BIBLIOGRAFÍA}

Alonso, J. \& Grande, I. (2004). Comportamiento del Consumidor. Madrid: ESIC.

Assael, H. (1987) Consumer Behavior and Marketing Action. 3rd Edition.

Beckman, H. W. \& Gilson, Ch. R. (1978) Consumer behaviour. Boston, Massachusetts: Rent Publishing, Co.

Benedict, R. (1934). Patterns of Culture. Nueva York: Houghton Mifflin, Co.

Bettman, J. R. \& Morgan, J. (1972) Formal models of consumer behaviour: a conceptual overview. En Journal of Business, 45 (4), pp. 544-562.

Bryant, J. \& Miron, D. (2002) Entertainment as media effect. En Bryant, J. \& Zillman, D. (2002) Media effects: Advances in theory and research. Pp. 549-582. Mahwah. 
Campos, F. (2008) Las redes sociales trastocan los modelos de los medios de comunicación tradicionales. En Revista Latina de Comunicación Social, núm. 63, pp. 287-293.

Cepeda, A. (2005) El comportamiento del consumidor de cine: una revisión preliminar de la literatura. En Revista Colombiana de Psicología, núm. 174, pp. 89-99.

De Borja, L., Casanovas, J. A. \& Bosch, R. (2002) El consumidor turístico. Madrid: ESIC.

Del Río, P., Álvarez, A. \& Del Río, M. (2004) Pigmalión. Informe sobre el impacto de la televisión en la infancia. Madrid: Fundación Infancia y Aprendizaje.

Eliashberg, J. \& Sawhney, M. S. (1994) Modeling goes to Hollywood: Predicting individual differences in movie enjoyment. En Management Science, 40 (9), pp. 11511173.

Eliashberg, J. \& Shugan, S. M. (1997) Film critics: Influencers predictors? En Journal of Marketing, 61, pp. 68-78.

Foxall, G. R., Goldsmith, R. E. \& Brown, S. (2002) Consumer Psychology for Marketing. 2nd Edition. Oxford: Thomson Learning.

França, M. E. (2001) La contribución de las series de televisión a la formación de la identidad de los adolescentes. Tesis doctoral. Universidad Autónoma de Barcelona.

Frank, R. E., Massy, W. F. \& Wind, Y. (1972) Market segmentation. Englewood Cliffs, NJ: Prentice-Hall.

Gardner, M. P. (1985) Mood states and consumer behavior: A critical review. En Journal of Consumer Research, 12 (3), pp. 281-300.

Greenberg, B., Rampoldi, H. \& Ver, S. L. (1998) Young viewers responses to television program ratings. Paper presentado a The Mass Communication Division, International Communication Association, Jerusalem, Israel.

Hirschman, E. C. (1987) Consumer of preferences in literature, motion pictures, and television programs. En Empirical Studies of the Arts, 5 (1), pp. 31-46.

Holbrook, M. B. \& Gardner, M. P. (2000) Illustrating a dynamic model of the mood uptadting process in consumer behavior. En Psychology and Marketing, 17 (3), pp. 165194.

Horton, J. \& Arquette, C. (2000) The role of television programming on secondary students self identity. New Orleans: Annual Meeting of the American Educational Research Association (AERA). 
Howard, J. A. \& Sheth, J. N. (1969) The Theory of Buyer Behavior. New York: Wiley.

Isen, A. M. (1984) Toward understanding the role of affect in cognition. En Wyer, W., Srull \& Isen, A. Handbook of social cognition, pp. 179-236. Lawrence eErlbaum.

Kotler, P. (2000) Dirección de Marketing. Herdfordshire: Prentice-Hall International.

Kretch, D. \& Crutchield, R. S. (1952) Theorie et Problemes de Psychologie Social. Press. Universitaires de France.

León, J. L. \& Olabarría, E. (1991) Conducta del Consumidor y Marketing. Ediciones Deusto.

Linton, R. (1968) The Concept of Culture. En Kassarjian, H. \& Robertson, T. (Eds.) Perspectives in Consumer Behavior.

Linton, J. M. \& Petrovich, J. A. (1988) The application of the consumer information acquisition approach to movie selection: An exploratory study. En Austin, B. A. (Ed.) Current research in film: Audiences, economics and law, vol. 4, pp. 24-44. Norwood, NJ: Ablex.

López, N. (2009) Preferencia juvenil en nuevos formatos de televisión: tendencias de consumo en jóvenes de 14 a 25 años. Proyecto de investigación financiado por Observatorio del ocio y el entretenimiento digital, OCENDI-Lúdico (NEO Comunicación Profesional, SL.). Periodo 2009-2011. Cód. OCENDI010911.

Mishra, V. (2009) Spectres of Sentimentality: the Bollybood Film. Textual Practice, 23:3, pp. 439-462.

Medrano, C., et al. (2010) Los programas y características de los personajes preferidos en el visionado de la televisión: diferencias evolutivas y de sexo. En Cultura y Educación, 22 (1), pp. 3-20.

Mestre, V. et al. (1998) Diferencias de género en la empatía y su relación con el pensamiento moral y el altruismo. IberPsicología, 3 (1).

Miller, L. E. \& Grush, J. E. (1986) Individual Differences in Attitudinal Versus Normative Determination of Behavior. En Journal of Experimental Social Psychology, vol. 22, pp.190-202.

Mollá, A. (coord) et al. (2006) Comportamiento del consumidor. Barcelona: Editorial UOC.

Montero, Y. (2006) Televisión, valores y adolescencia. Barcelona: Gedisa. 
Mustonen, A. \& Pulkinen, L. (1997) Television violence: A development of a coding schema. En Journal of Broadcasting and Electronic Media, 41 (2), pp. 168-189.

Nicosia, F. (1966) Consumer Decision Processes: Marketing and Advertising Implications. Prentice Hall.

Olivenr, M. B. (2002) Individual differences in media effects. En Bryant, J. \& Zillman, D. Media effects: Advances in theory and research, pp. 507-524. New Jersey: Lawrence Erlbaum.

Oliver, M. B. (2008) Tender affective states as predictors of entertainment preference, Journal of Communications, 58, pp. 40-61.

Palmgreen, P. \& Lawrence, P. A. (1991) Avoidances, gratification, and comsuption of theatrical films: The rest of the story. En Austin, B. A. Current research in film: Audiences, economics and law, vol. 5, pp. 39-55. Norwood, NJ: Ablex.

Ryan, R. M., Rigby, C. S. \& Przybylski, A. (2006) The motivacional pull of video games: A self-determination theory approach. En Motivation and Emotion, 30 (4), pp. 347-363.

Sánchez-Queija, J., Oliva, A. \& Parra, A. (2006) Empatía y conducta prosocial durante la adolescencia. En Revista de Psicología Social, 21 (3), pp. 259-280.

Schnake, H. (1988) El Comportamiento del Consumidor. Ed. Trillas.

Seiter, E. (1989) A Remate Control: Television Audiences and Cultural Power. Londres: Routege.

Sherif, M. \& Cantril, H. (1974) The Psychology of Ego-Involvement. New York: John Wiley and Sons.

Sherry, J. F. (1986) The Cultural Perspective in Consumer Research. En Lutz, R. J. (Ed.) Advances in Consumer Research, vol. 13, pp. 573-575.

Tylor (1913) Primitive Culture. Murray.

Vaca, R. (2010) El perfil de la audiencia. Cuatro y la Sexta, 2005-2008. Fundación ExLibris y Barlovento Comunicación.

Veitch, R. \& Griffitt, W. (1976) Good news-bad news: Affective and interpersonal effects. En Journal of Applied Social Psychology, 6 (1), pp. 69-75.

Watt, J. \& Krull, R. (1977) An examination of three models of television viewing and aggression. En Human Communication Research, 3 (2), pp. 99-112. 
Wilkie, W. L. (1994) Consumer behavior. New York: Wilie \& Sons.

Winoto, P. \& Tang, T. (2010) The role of user mood in movie recommendations. En: Expert Systems with Applications, núm. 37, pp. 6086-6092.

Zillman, D. (1988) Mood management: Using entertainment to full advantage. En Donohew, L., Sypher, H. E. \& Higgings, E. T. Communication, social cognition, and affect. Hillsdale, NJ: Lawrence Erlbaum. Pp. 147-171.

\section{Noelia Araújo Vila.}

Licenciada en Administración y Dirección de Empresas (2006) y actualmente está realizando el Doctorado en Dirección y Planificación del Turismo (Universidad de Vigo). Desde el año 2007 ha colaborado con la Universidad de Vigo impartiendo docencia y participando en proyectos de investigación del ámbito turístico y empresarial, así como cooperando en la coordinación del Máster Universitario en Dirección y Planificación del Turismo.

\section{José Antonio Fraiz Brea}

Doctor en Ciencias Económicas y Empresariales (1995) y desde el año 1997 profesor titular en la Universidad de Vigo. En su trayectoria profesional son numerosas las publicaciones y colaboraciones en proyectos de investigación vinculados con el sector empresarial y turístico. Entre las numerosas tareas de gestión y coordinación que ha desarrollado, destaca el cargo de director del Master Universitario en Dirección y Planificación del Turismo (Universidad de Vigo) desde el año 2006, año de inicio del mismo. 